\title{
COMMENTARY: ESOPHAGEAL CARCINOMA CONFINED TO THE WALL-THE NEED FOR IMMEDIATE DEFINITIVE THERAPY
}

Thomas W. Rice, MD

$C^{3}$ arcinoma of the esophagus and esophagogastric junction is a lethal disease. Our ability to cure this cancer is limited because most patients have dysphagia and advanced stage disease when first evaluated. A symptomatic tumor that obstructs the esophageal lumen has usually spread beyond the esophageal musculature to involve the periesophageal tissue and/or adjacent organs and has metastasized to regional lymph nodes and/or distant sites.

The recent shift in epidemiology of esophageal carcinoma provides an opportunity for improved survival. This epidemic of adenocarcinoma in middle-aged and elderly white men permits screening of a high-risk group, which can identify patients with carcinomas

From the Department of Thoracic and Cardiovascular Surgery, The Cleveland Clinic Foundation, Cleveland, Ohio.

Requested for publication Aug 5, 1998; received Sept 21, 1998; accepted for publication Oct 1, 1998.

Address for reprints: Thomas W. Rice, MD, Head of the Section of General Thoracic Surgery, The Cleveland Clinic Foundation, 9500 Euclid Ave, Cleveland, OH 44195.

J Thorac Cardiovasc Surg 1999;117:26-7

Copyright $\odot 1999$ by Mosby, Inc.

$0022-5223 / 99 \$ 8.00+0 \quad \mathbf{1 2 / 6 / 9 4 9 8 0}$

confined to the esophageal wall. However, a carcinoma confined to the esophageal wall is not necessarily a carcinoma that has not metastasized.

Lymphatic drainage of the esophagus allows for early dissemination of carcinoma in many patients before symptoms develop (Fig 1). The presence of lymphatics in the lamina propria and muscularis mucosa deep to the basement membrane makes the esophagus unique among gastrointestinal hollow viscus organs. These lymphatics drain into the rich submucosal lymphatic plexus and spread longitudinally through this dense lymphatic network. Lymphatics intermittently pierce the muscularis propria to drain into regional lymph nodes or directly into the thoracic duct. This anatomic peculiarity exposes lymphatics to carcinoma at the earliest stage of invasion, just after the carcinoma breaches the basement membrane and invades the lamina propria (Fig 2). As tumors invade deeper into the wall, there is rapid, extensive spread in the submucosal lymphatic network and an increasing prevalence of regional lymph node metastases. ${ }^{1}$

Nigro and colleagues ${ }^{2}$ report a prevalence of lymph node metastases that increases with increasing depth of tumor invasion. The prevalence of regional lymph node

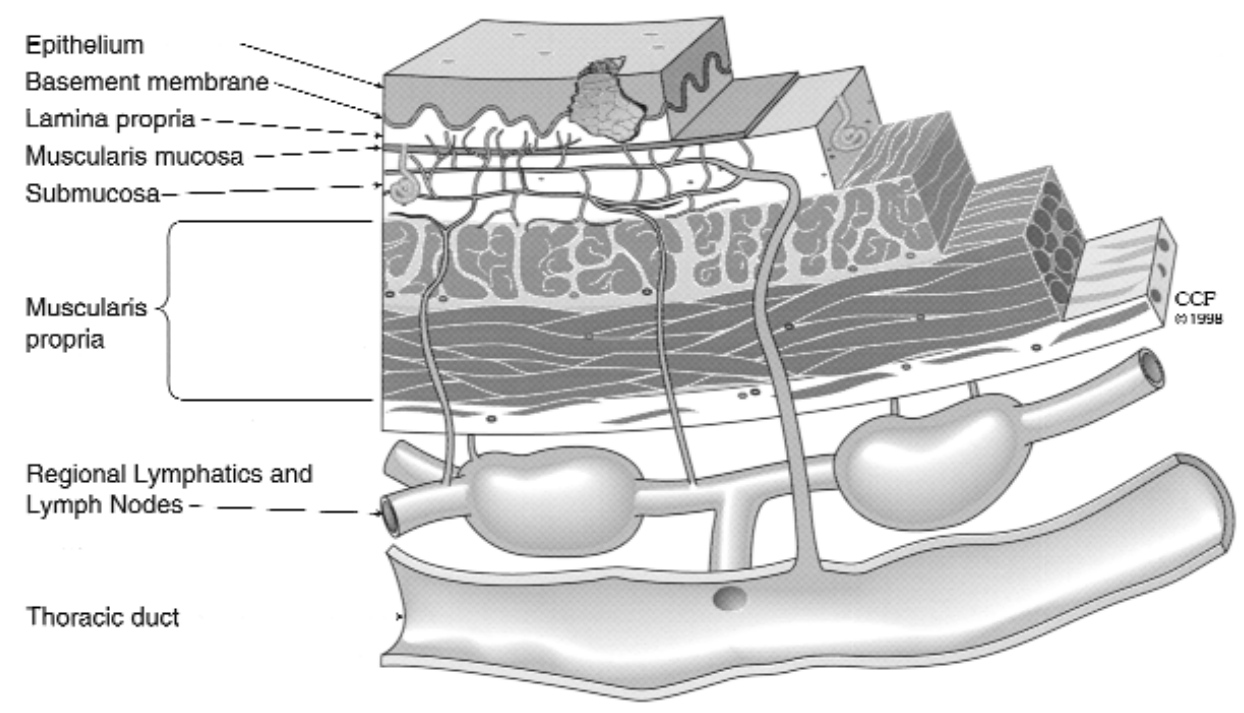

Fig 1. The lymphatic anatomy of the esophagus. Lymphatics are found in the lamina propria just below the basement membrane. An intramucosal tumor is seen with invasion of the lamina propria. 
metastases (N1) was 7\% for T1 tumors with invasion confined to the esophageal mucosa (lamina propria or muscularis mucosa), $50 \%$ for T1 tumors with submucosal invasion, and $80 \%$ for T2 tumors. Distant nodal metastases (M1a) were detected in $14 \%(1 / 7)$ of patients with $\mathrm{T} 1$ carcinomas and $63 \%$ (5/8) of patients with $\mathrm{T} 2$ tumors. These findings are consistent with the lymphatic anatomy of the esophagus where deeper invasion is associated with increasing lymphatic exposure and therefore an escalating potential for both regional and distant lymph node metastases.

Cure is possible only if a carcinoma is contained in the treatment field. In patients with high-grade dysplasia (intraepithelial carcinoma), there is no chance for lymphatic spread because the malignant tumor is limited by the basement membrane., ${ }^{1,3,4}$ The potential of cure decreases exponentially with increasing invasion of the esophageal wall.

Mucosal ablative treatments (photodynamic therapy) are dangerous. These experimental therapies incompletely destroy the superficial layers of the esophageal wall and do not penetrate to eradicate deeper invasion or submucosal lymphatic spread. The dysplastic or invasive changes may be submerged below the pseudosquamous epithelium, thus hiding these changes from surveillance. Most importantly, curative treatment is delayed, allowing deeper invasion and lymphatic metastases.

Diligent surveillance of high-risk groups identifies patients with preinvasive or early invasive carcinomas. Excision before lymphatic invasion is the only hope for improved survival.

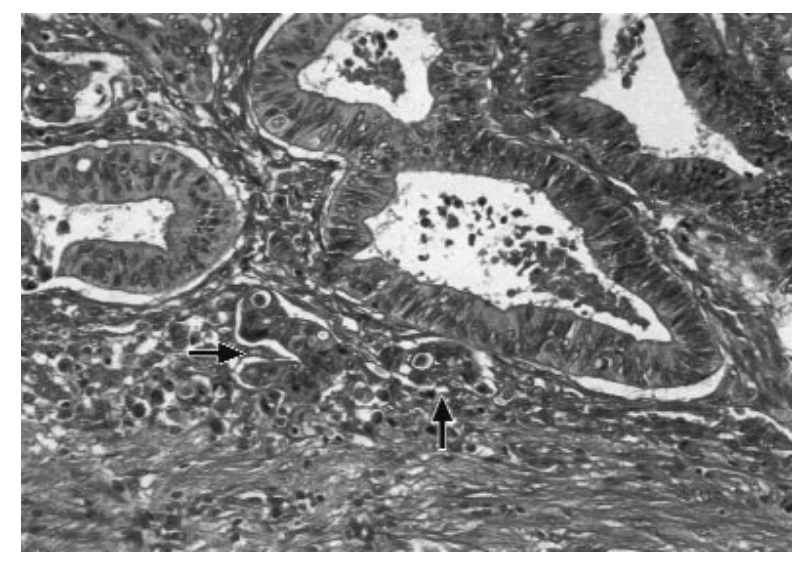

Fig 2. Photomicrograph of intramucosal adenocarcinoma of the columnar-lined esophagus with invasion of the lymphatic in the lamina propria (arrows).

\section{REFERENCES}

1. Rice TW, Zuccaro G Jr, Adelstein DJ, et al. Depth of tumor invasion $(\mathrm{T})$ is predictive of regional lymph node status $(\mathrm{N})$ for esophageal carcinoma. Ann Thorac Surg 1998;65:787-92.

2. Nigro JJ, Hagen JA, DeMeester TR, et al. Prevalence and location of nodal metastases in distal esophageal adenocarcinoma confined to the wall: implications for therapy. J Thorac Cardiovasc Surg 1999;117:16-25.

3. Rice TW, Falk GW, Achkar E, Petras RE. Surgical management of high-grade dysplasia in Barrett's esophagus. Am J Gastroenterol 1993;88:1832-6.

4. Sabik JF, Rice TW, Goldblum JR, et al. Superficial esophageal carcinoma. Ann Thorac Surg 1995;60:896-902. 